\title{
ELECTRONIC SPECTRA OF ORGANIC SOLID SOLUTIONS: EFFECTS OF LATTICE DISORDER ON SPECTRAL LINE WIDTHS AND MULTIPLICITIES
}

\author{
SYDNEY LEACH \\ Laboratoire de Photophysique Moléculaire†, Bâtiment 213, \\ Faculté des Sciences, 91-Orsay, France
}

\begin{abstract}
Line widths and multiplicities observed in solute electronic spectra of dilute organic solid solutions depend to a great extent on lattice disorder and solute site inhomogeneities. A definition of the structure of different types of organic solids is followed by a discussion on defects in these solids and of some of their photophysical consequences. The formation of organic solid solutions and the effect of various thermal treatments are considered in the light of the previous discussion. The influence of lattice phonon structure on spectral line widths is discussed for near-perfect and increasingly disordered solid solutions, in terms of the optical analogue of the Mössbauer effect. At low temperature effect is less important than static and dynamic solute site inhomogeneities in determining observed spectral line widths. The latter should be narrowest for substitutional solutions. The formation of such solutions depends on a number of parameters amongst which are the solute and solvent molecular geometries and volumes. The effect of these two parameters on solute spectral line widths has been studied. Multiplet structure in the electronic spectra of crystalline organic solutions is discussed. The origin of solute site plurality is considere in terms of the mechanism of crystal growth.
\end{abstract}

\section{INTRODUCTION}

The electronic and geometrical structures and the vibrational dynamics of organic molecules can be determined by detailed analysis of the electronic spectra of these species in the gas phase at low pressures. In the solid phase, molecular spectra will be perturbed to an extent that depends on the nature and the degree of intermolecular coupling. The electronic spectra of organic solid solutions have been used to characterize the spectroscopic and structural properties of solute molecules, in particular under experimental conditions where the solute molecules are essentially isolated in suitable matrices at low temperatures. These conditions are particularly favourable for the study of the optical transitions of trapped free radicals and the phosphorescence from triplet states of aromatic stable molecules. Furthermore, in

$\dagger$ Groupe de recherche du C.N.R.S. associé à la Faculté des Sciences d'Orsay. 
matrices ate low temperatures, hot bands are generally suppressed thus facilitating vibronic analysis ${ }^{1,2}$.

The perturbation of the solid solution spectra with respect to the gas phase can be used to study certain structural and dynamic properties of the matrix and also to determine the state of dispersion and aggregation of the solute ${ }^{1,3}$.

Among other applications of electronic spectroscopy of organic solutions, particularly pertinent to the present symposium, are the study of energy transfer ${ }^{4}$ and photochemistry in the solid state ${ }^{5}$.

Most of the earlier spectroscopic work on organic solid solutions, from 1910 to 1960 , was done in glassy matrices at low temperatures. Particular mention should be made to the pioneer work of von Kowalski ${ }^{6}$, on stable molecule solutes, and to the studies of Lewis and his co-workers ${ }^{7}$ and Porter and his co-workers ${ }^{8}$ on the absorption spectra of aromatic radicals trapped in rigid organic glasses. Fluorescence spectra of trapped radicals have also been studied in rigid glass solutions ${ }^{5 \mathrm{a}, 9}$.

In $1954 \mathrm{McClure}^{10}$ introduced the 'oriented gas' type of study in which the solute is presumed to be in a substitutional site in an aromatic crystalline matrix. Two years earlier, Shpolskii ${ }^{11}$ observed narrow line fluorescence spectra of aromatic solutes in crystalline linear paraffin matrices and has since created a very active school which has made a considerable number of spectroscopic studies using the 'Shpolskii effect'12.

However, much of the published work on the electronic spectra of organic solid solutions is difficult to interpret without ambiguity since many insufficiently studied parameters govern their structure. These parameters include: (a) modification of the intrinsic electronic and structural properties of the solute molecule with respect to the gas phase; (b) the state of dispersion of the solute molecules; (c) the nature and configuration of the solute site; (d) solute-solute interactions; (e) solute-matrix interactions. Furthermore, these parameters are often sensitive to a number of experimental variables, not always well controlled or indicated in earlier publications, such as the method of preparation of the solid solutions and their thermal history.

A systematic discussion has recently been made concerning some of the above parameters ${ }^{1,3}$. The present paper can be considered as a development of certain aspects of references 1 and 3 . In it will be discussed problems concerning spectroscopic line widths and multiplet structure which were only briefly mentioned in the previous study or which were considered from a different viewpoint. Their treatment here requires an initial discussion, which is given in the next section, of the basic physics of organic solids and solutions relevant to electronic spectroscopy.

\section{THE STRUCTURE OF ORGANIC SOLIDS}

\section{1. Crystals and amorphous solids}

Spectroscopists and photochemists studying organic solids usually classify them as belonging to one of three categories: monocrystals, polycrystalline material and glasses. In molecular solids composed of organic molecules the electrostatic and dispersive intermolecular attractive forces are of the 
order of a few $\mathrm{kcal} / \mathrm{mole}$. The particular molecular arrangement in such solids will be determined essentially, by a true or a metastable minimum of free energy.

For organic crystals the criterion of minimum free energy will usually result in a tendency to form a close packed structure ${ }^{13}$ whose particular configuration will depend mainly on the repulsive forces between the peripheral atoms of neighbouring molecules ${ }^{14,15}$.

If several close packed structures of similar energies are possible, there will be a tendency for the crystal to assume the structure having the highest number of symmetry elements ${ }^{14}$.

Monocrystals are characterized by a lattice structure in which the constituent molecules are related by periodic translations. Long range order exists over a large, virtually infinite number of lattice cells in an ideal monocrystal. A single crystal is an individual monocrystal.

Polycrystalline material is composed of a mosaic of monocrystals having a variety of relative spatial orientations. In the ideal case, long range order for each monocrystal ceases at the mosaic limits. The existence of manifold crystal boundaries gives rise to high scattering of visible light and an opaque quality as compared to the low light scattering and, in appropriate spectral regions, the transparency of single crystals.

The existence of long range order is testified by $x$-ray and electron diffraction studies of single crystals and polycrystals. When the regions of organized structure are non-existent or small (for example, periodicity no greater than $50-100 \AA$ ) and the grain boundaries are very extensive, then the solid can be said to have an amorphous structure and is highly opaque. A glass is another form of amorphous structure in which periodicity does not exist or only over a very small region, but for which there is apparently no granular structure so that the glass is transparent.

Very little is known about the structure of amorphous solids and glasses composed of organic materials except for polymers ${ }^{16}$.

\section{2. Defects in organic solids}

Spectroscopic, photochemical and energy transfer properties of perfect organic solids will be modified in the presence of intrinsic or extrinsic (impurity) defects.

Thermodynamic considerations show that defects will always be present in solids. Consider, for example, the formation of a vacancy in a crystal. It should first be remarked that the molecular diffusion concomitant with vacancy creation is theoretically possible at any temperature since the crystal always contains internal vibrational energy. Vacancy creation will increase the crystal energy since energy has to be expended in overcoming the forces which tend to keep a molecule in its initial position in the lattice. However, at the same time there will also be an increase in the entropy of the system since some configurational order is destroyed in the crystal. A theoretical equilibrium concentration of vacancies, greater than zero, will therefore exist at each temperature. The value of this equilibrium concentration will depend on the compromise between the principles of minimum energy and maximum entropy. A similar discussion can be developed for other types of defects ${ }^{17}$. 
Although experimental investigations of defects have been extensive for many types of solids, it is only within recent years that such studies have been undertaken for organic solids ${ }^{18}$. Data on organic solids are still rather limited, so that part of the following discussion is based on information obtained on other systems.

In organic crystals, the intrinsic point defects will be, in general, lattice vacancies. Interstitial defects are not likely in closed packed structures. Another type of point defect can arise from the presence of disoriented molecules in the lattice. Linear dislocations of the edge and spiral types, and planar dislocations of the grain boundary and twinning types also occur. The planar dislocations can be considered as creating internal surfaces in crystals. Another type of internal boundary occurs when crystal grains of different crystallographic order or of different chemical composition exist juxtaposed.

Radiotracer and other studies of self-diffusion (point defect migration) have been made in organic crystals ${ }^{18}$. Rates of self-diffusion along dislocations and sub-grain boundaries are higher than for self-diffusion through the lattice. Intrinsic self-diffusion rates are $10^{4}$ times greater for plastic crystals, such as the high temperature form of cyclohexane, than for linear condensed aromatic crystals. For benzene, the point defect diffusion rate is about $10^{2}$ times lower than for cyclohexane. The large differences in defect mobilities in different systems will be significant in determining the defect concentration in organic solid solutions.

Another important factor concerns dislocation glides in which there is a slip of adjacent crystal planes in the neighbourhood of a dislocation. As far as a particular slip system is concerned, these glides may be different for pure crystals and for crystals containing impurity molecules.

It should be noted that impurity molecules can be substitutional defects or, if they are of sufficiently small dimensions, they can occupy interstitial positions. When the impurity molecule has a much larger volume than the matrix molecules, more than one matrix molecule can be displaced or replaced.

One can also envisage a number of defect complexes ${ }^{17}$ : impurity moleculevacancy pairs; vacancy clusters (although these should be rare in annealed close packed molecular solids); dislocation-vacancy interactions; dislocationimpurity molecule complexes.

In addition, impurity-impurity complexes, leading eventually to solute aggregation can occur in many systems. and can be detected spectroscopically ${ }^{1,3}$. In the discussion in this paper we will leave aside the problem of solute aggregation which has been dealt with elsewhere ${ }^{1,3}$.

Defects and defect complexes will have specific spectroscopic, photophysical and photochemical consequences. For organic crystals, they will tend to act as traps limiting or modifying exciton and phonon propagation in the matrix. The trapping properties will depend on the interaction between the defect electronic or vibrational states and these quasi-particles. The extent of exciton or phonon trapping will be temperature dependent. Critical defect concentrations and temperatures for these effects will depend not only on the type of defect or defect complex but also on the particular exciton-for example, singlet or triplet-or phonon wave packet. It is 
therefore to be expected that in organic solid solutions, the luminescence spectra (quantum yield ${ }^{19}$ and spectral structure ${ }^{20}$ ) of the matrix and of the solute and the yield of matrix $\rightarrow$ solute energy transfer will be functions of the nature and concentration of defects as well as of the temperature ${ }^{21}$.

\section{3. The formation of organic solid solutions}

Spectroscopic studies on organic solid solutions have been done mainly on solid solutions prepared from the melt or liquid phase and subjected to different types of cooling procedures.

\section{(a) Glasses}

Glass preparation is usually done by low temperature quenching of the glass forming liquid. Little data exist on the physics of the formation of organic glasses. However, from information on polymer and inorganic glasses it is reasonable to suppose that in glass-forming organic compounds the high viscosity ( $\sim 10^{12}$ poise) at the liquidus temperature prevents ordered alignment of the constituent molecules. A high degree of configurational order will therefore not occur on freezing.

The arrangement of the constituent molecules in the glass will be similar to the 'instantaneous' structure of the liquid. Local ordering may or may not occur, according to the presence or absence of ordered cybotactic groups in the liquid phase. Such cybotactic groups could not be of large extent in glass-forming materials otherwise it would be difficult to account for the high viscosity and diffusion properties in the region of the liquidus temperature. Furthermore, if order is present in local domains in the glass its configuration does not necessarily correspond to that of a crystalline variety of the same substance. The existence of so-called short-range order in liquids or glasses as determined by diffraction or scattering techniques usually gives little information as to the existence of any true periodic structure since the correlation functions obtained by these methods often simply reflect a statistical distribution of intermolecular distances and are so hardly informative about true local order.

Many different types of solvent and solvent mixtures have been used to form organic glasses ${ }^{22}$ for electronic spectroscopy and organic solid-state photochemistry. Organic glasses will tend to form if the constituent molecules are associated in the liquid through hydrogen bonding, which therefore opposes certain forms of ordered alignment, or if the solvent molecules contain a multiplicity of side chains and so possess many quasi-independent degrees of local movement.

(b) Crystalline solid solutions

Rapid quenching, slow cooling, and rapid quenching followed by annealing at a fixed temperature, are the three thermal treatments usually employed in forming crystalline solid solutions from the liquid phase. These different techniques can give rise to samples having substantially different spectroscopic and photochemical properties since the crystallographic structure is sometimes, and the defect structure and concentration are always, dependent on the thermal history of the sample ${ }^{4,23}$. Furthermore, the presence of occluded gases can have important perturbing effects on the structure of the solid formed ${ }^{4}$. 
The following model is proposed concerning the structure of the matrix in the case of quenching. The liquid phase may contain cybotactic or paracrystalline domains, or these may be absent. On rapid freezing there will be dissipation of heat from the solvent, this dissipation generally being anisotropic in quenching experiments. Nucleation rapidly takes place leading to an incipient microcrystal which could have a faulted structure initially of lower free energy than a non-faulted structure (see section V). Rapid growth of the microcrystal will occur, leading to the introduction of a large number of defects. However, the continual lowering of the temperature tends to build up high strain energy since the barrier to self-diffusion will increase as thermal energy is reduced. Strain relief will occur by cleavage and by the introduction of dislocations. The fact that organic crystals have high thermal expansivities also contributes to the formation of lattice strain. The sample will therefore tend to consist of a mass of small crystallites of high defect content.

Occluded gases will tend to be trapped and act as extrinsic defects further perturbing the regularity of the polycrystal lattices and leading to dislocation and microcrystal formation. It is to be emphasized that the defect concentration at the quench temperature $T_{q}$ will be very much greater than the equilibrium concentration would have been at $T_{q}$.

The solute molecules (neglecting solute aggregation) will tend to be fixed in a variety of defect and defect-complex situations. This will lead to spectral line broadening or multiplet structure according to the statistical distribution of solute-matrix interactions, as will later be discussed in detail.

In the case of slow cooling (and leaving aside the question of the possibility of considerable supercooling) there will exist at the freezing point $T_{f}$ a certain defect structure in the nucleated growing crystal whose concentration will correspond approximately to that of the equilibrium concentration at $T_{f}$. Let us assume extremely slow cooling. As the temperature is very gradually lowered there will be a decrease in the equilibrium number of intrinsic point defects. Here too, there will be a tendency for lattice strain energy due to excess point defects to be relieved by their migration (at a rate depending on the self-diffusion rate) to form dislocations, and eventually by cleavage. However, slow cooling should lead to a smaller dislocation content and to larger single crystals than rapid cooling since a process of continuous annealing will tend to occur in the former case.

Nevertheless, on practical time scales for laboratory crystal growth, the true concentration of defects will in general be greater, and often considerably greater, than the equilibrium concentration since at very low temperatures the diffusion rate will be extremely slow. It is important to note the importance of the remark made in the previous section, that plastic crystals, e.g. the high temperature form of cyclohexane, have much higher self-diffusion rates than 'brittle' crystals such as the linear condensed aromatic molecules. One might therefore expect plastic crystals to have a much smaller defect concentration than the 'brittle' crystals. This could lead to a much smaller statistical width of solute-matrix interactions in the case of plastic crystal matrices and could so give rise to narrower spectral' line widths than for 'brittle' crystal matrices (although as we shall see, the relative size of the solute and solvent molecules is also a capital factor). 


\section{ELECTRONIC SPECTRA OF ORGANIC SOLID SOLUTIONS}

In some experiments in our laboratory, solid crystalline solutions have been formed by annealing rapidly quenched samples, the annealing being carried out at a temperature just below the freezing point or close to a crystallographic phase transition temperature. J. Kahane-Paillous and C. Pfister have observed that the spectra of coronene solutions in $n$-heptane, are identical after slow cooling and after annealing if the latter is carried out for 2 or 3 days $^{25}$. In our laboratory, C. Michel found for the anthracene- $n$ heptane system that 20 hours annealing just below the freezing point sufficed to obtain the same spectral behaviour as a slowly cooled solution (one hour from $300^{\circ} \mathrm{K}$ to $\left.80^{\circ} \mathrm{K}\right)^{26}$.

One further point should be noted. Molecular solids, in particular plastic crystals, usually possess several crystallographic forms and thus exhibit crystallographic phase transitions at certain temperatures. The plastic is often a high temperature form that goes over to a 'brittle' form at a phase transition temperature thus creating new stresses and giving rise to cleavage of a monocrystal initially in the plastic form. This makes it difficult to prepare monocrystals for spectroscopic studies in the stable low temperature crystallographic forms of crystals of molecules such as cyclohexane.

\section{SPECTROSCOPIC LINE WIDTHS IN ORGANIC SOLID SOLUTIONS: INFLUENCE OF THE PHONON STRUCTURE}

Molecular rotation is virtually quenched in low temperature solids and so does not contribute to vibronic band widths. The principal factors governing widths of solute vibronic bands of an organic solid solution at a particular temperature are the phonon structure of the lattice and the site inhomogeneity of the solute. The influence of the phonon structure will first be examined. Interpretations of the narrow line widths observed in organic crystalline solid solutions (Shpolskii effect) have been made in recent years in terms of an optical analogue of the Mössbauer effect and are therefore based essentially on the phonon structure and phonon relaxation phenomena in the solid ${ }^{27}$.

Let us consider the phonon structure of an ideal organic crystal and its relation to the width of solute vibronic bands and then examine the effects of imperfections and disordered structure on the band widths.

It will first be assumed that solute concentration is sufficiently low, and the solute molecules sufficiently well dispersed, for solute-solute interactions to be negligible ${ }^{1,3}$. Furthermore it is assumed, throughout this paper, that the energy of the electronic excited state in the solute optical transition under consideration is more than $1 \mathrm{eV}$ below the lowest exciton level of the matrix, so that mixing of solute and matrix excited states will be unimportant in determining solute spectral line widths ${ }^{3}$.

\section{1. Nearly perfect crystals}

The atomic displacements in the crystal can be resolved into lattice waves at any instant. The lattice waves will be a superposition of normal modes of the lattice, the energy content of each mode being time variant. In the nearly perfect crystal, each lattice wave will be composed chiefly of one 
particular lattice vibration mode. Interchange among modes, i.e. the variation of energy content among the superposed normal modes, will be slow and exists, on the harmonic model, only because of deviations from crystal perfection.

The presence of a solute molecule, i.e. an extrinsic impurity centre, will modify the strict periodicity in the equilibrium spatial positions of the molecular motifs in the crystal. The solute molecules, and a neighbouring region of matrix molecules where lattice translational symmetry is effectively lost, will constitute a perturbed local region with particular properties concerning dynamic processes in the crystal in particular lattice vibration relaxation processes.

Three types of vibrations are of importance for electronic spectroscopy of the solute molecule:

(1) Solute intramolecular vibrations which in general will be only weakly coupled to the matrix lattice since the interatomic forces within the molecule are of two orders of magnitude greater than intermolecular forces. Intramolecular vibrational modes will therefore manifest themselves in the electronic spectra virtually as if the solute molecule were isolated (i.e. similar to low pressure gas phase conditions) ${ }^{1,3}$.

(2) Localized vibrations of the solute which will essentially be librational and translational oscillations of the solute molecule in an apparent fixed cage of the matrix ${ }^{3}$.

(3) Nonlocalized vibrations which correspond to vibrations of the crystal

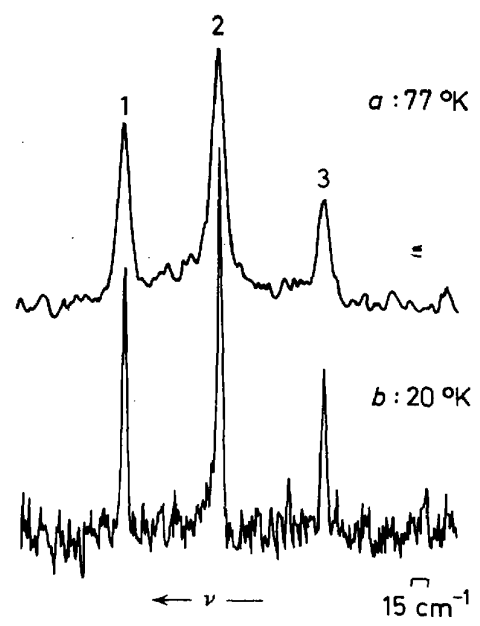

Figure I. Part of the fluorescence spectrum ${ }^{1} B_{2 \vec{u}} A_{1 g}$ of benzene in annealed solid solution in cyclohexane (concentration $10^{-3} \mathrm{M} / 1$ ). (a) $77^{\circ} \mathrm{K}$; (b) $20^{\circ} \mathrm{K}$.

Vibronic transitions ${ }^{52,53}: 1=0,0-9 \dot{9} 1\left(a_{1 q}\right) \quad\left(36722 \mathrm{~cm}^{-1}\right)$

$2=0,0-675\left(a_{2 u}\right)-402\left(e_{2 u}\right)\left(36636 \mathrm{~cm}^{-1}\right)$

$$
3=0,0-1173\left(e_{2 g}\right) \quad\left(36540 \mathrm{~cm}^{-1}\right)
$$

Spectrum $a$ has beerr displaced relative to spectrum $b$ to eliminate a temperature dependent shift of the spectral origin. 
lattice as a whole and which are essentially identical to the lattice vibrations of the matrix in the absence of solute inclusions ${ }^{3,28}$.

One might expect that vibronic transitions of the solute should show a fairly broad structure corresponding to lattice phonon creation or annihilation associated with the vibronic transition. However, Trifonov, Rebane and others $^{27}$ have shown that there is an optical analogue to the Mössbauer effect in which the $\gamma$-ray recoil kinetic energy is replaced by the excess potential energy created by the difference in intermolecular interactions in the ground and excited states. In similar fashion to the Mössbauer case there is a high probability for transitions to occur in which no phonons are created or destroyed (i.e. $\Delta n=0$ transitions, where $n$ is a quantum number associated with nonlocalized lattice vibrations). These are the so called zerophonon transitions. In the approximation of harmonic vibrations and identical oscillators in the excited and unexcited lattice, the no-phonon transitions corresponding to a particular vibronic band will coalesce to give a spectral line whose width should be of the order of $10^{-3} \mathrm{~cm}^{-1}$ for an allowed transition and even smaller for a forbidden transition. At the same time there will exist a background or wing corresponding to the creation and annihilation of phonons, i.e. transitions where $\Delta n>0$, whose shape will therefore also depend on the phonon population of the initial state at a given temperature. It is the spectral background, continuous because of the high density of phonon states, which manifests the relaxation, among the normal lattice modes, of the excess potential energy created by the optical transition.

On this model, an increase in temperature should not lead to a change in the zero-phonon line width but only to a modification of the relative intensity of the zero-phonon line and the associated continuum.

In a more refined treatment, dealt with at length in the recent book of Rebane $^{27}$, the consideration of anharmonicity, displaced oscillators and distorted oscillators, leads to an increase in zero-phonon line width due to thermal broadening, or, more precisely to a splitting of the $\Delta n$ transitions which at practical spectral resolution will be unresolved and give rise to a broadened line envelope. However, the line width, although increased by one or two orders of magnitude above the harmonic undisplaced oscillator case, will still be much smaller, on this refined model, than experimental line widths at low temperatures which are of the order of $1 \mathrm{~cm}^{-1}$ and greater for the zero-phonon lines ${ }^{1,24,27,29}$.

Although at low temperatures observed electronic spectra have broader lines than is expected only on the basis of the optical analogue of the Mössbauer effect, their shape and temperature behaviour show general qualitative agreement with the distorted and/or displaced oscillator model of this effect.

Figure 1 shows the widths of three typical bands of the fluorescence spectrum of benzene in cyclohexane $\left(10^{-3} \mathrm{M} / 1\right)$ at $20^{\circ} \mathrm{K}$ and at $77^{\circ} \mathrm{K}$. A background or wing due to phonon creation or annihilation processes is not very extensive in these spectra. The effect of increasing the temperature between $20^{\circ} \mathrm{K}$ and $77^{\circ} \mathrm{K}$ is mainly to broaden the line widths.

In Figure 2 is shown a part of the reflection spectrum of benzene in cyclohexane at temperatures between $80^{\circ} \mathrm{K}$ and $163^{\circ} \mathrm{K}$. At $80^{\circ} \mathrm{K}$, the 0,0 and the $0,0+521$ bands both exhibit a zero-phonon line and an extensive 


\section{SYDNEY LEACH}

phonon wing which is mainly to higher energies. The intensity variation observed within these wings indicate peaked phonon distributions, the maxima probably corresponding to localized vibrations.

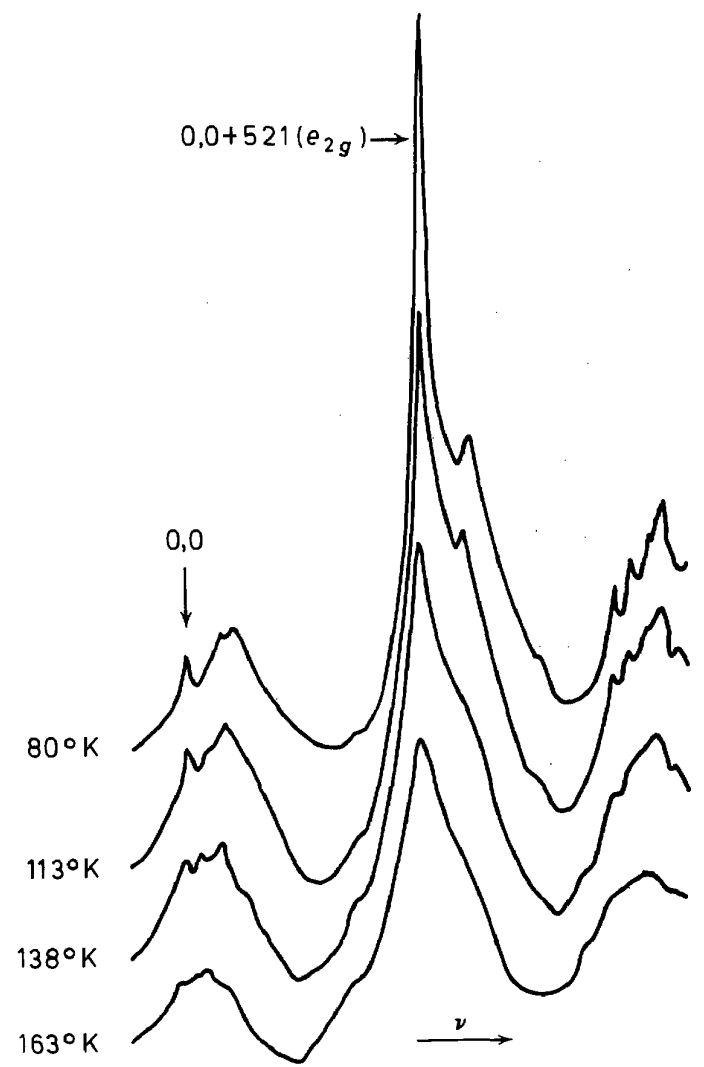

Figure 2. Part of the reflection spectrum ${ }^{1} B_{2 u}-A_{1 g}$ of benzene in solid solution in cyclohexane (concentration $10^{-3} \mathrm{M} / \mathrm{l}$ ) as a function of temperature. The temperature dependent shift of the spectral origin has been eliminated by relative displacement of the spectra.

As the temperature increases the zero-phonon lines progressively broaden and their maxima decrease in height. There is a concurrent increase in the integrated intensity of the phonon wings whilst the subsidiary maxima shift to lower energies.

It is to be remarked that the phonon wing associated with the 0,0 transition is more important than that associated with transitions involving excitation of intramolecular vibrations. This is probably related to the fact that the pure electronic transition is forbidden for the ${ }^{1} B_{2 u}{ }^{1} A_{1 g}$ transition of a $D_{6 h}$ benzene. Since the observed 0,0 band arises because of interactions with the crystal field, it will only appear weakly, but phonon assisted 0,0 transitions could become formally allowed through coupling of the pure electronic transition with lattice vibrations of appropriate symmetry and so give a relatively intense wing with respect to the zero-phonon 0,0 line. 
We have observed that phonon assisted transitions are generally more evident in absorption or reflection spectra than in fluorescence spectra. Since in the former spectra the phonon wing is essentially to higher energies with respect to the zero-phonon line, this result indicates that phonon creation has a greater probability in absorption to an excited electronic state than in fluorescence to the ground electronic state. In this connection it should be noted that the solute in its excited state will, in general, be located within a strained, electronically unrelaxed, solvent cage (see section III.3). The propensity for creation of phonons associated with the optical transition may thus be enhanced in the absorption/reflection spectra as compared with the fluorescence transition; in fluorescence, the relaxation of the solutesolvent cage complex can be effected, if necessary completely, through electronic redistribution in the solute molecule in going from the excited to the ground electronic state.

\section{2. Disordered solids}

The above discussion has been concerned with the phonon structure in the case of an assumed near perfect crystal, in which implicitly the solute molecules occupy substitutional sites in the lattice. We will now examine the effect of increasing disorder on the phonon structure in the solid. This disorder will be considered to be of two types, inhomogeneity in the sites of the solute molecules and intrinsic defect structures in the matrix. The most disordered structure will be that of an amorphous solid or a glass, as discussed in section II.

Although as disorder increases in the solid the periodic properties are progressively lost, it must be remembered that each molecular unit has an equilibrium site and vibrates about that site. Even an amorphous solid will have, in principle, normal modes of vibration. The solute intramolecular vibrations will hardly be modified by the increasing disorder in a molecular solid since their coupling with the matrix will in general remain weak. As far as the matrix is concerned the nonlocalized vibrations will have a frequency spectrum of normal modes which differs little from that of the lattice waves of the perfect crystal except in the high frequency region which describes the relative motion of neighbouring molecular units. Indeed the normal modes of low frequency will be similar in character to the waves in a homogeneous isotropic elastic continuum, for in the limit of long waves the atomic structure is unimportant.

As disorder increases, the phonon relaxation time will decrease, i.e. the rate of interchange of energy among the normal modes will become more rapid. As the limit of an amorphous solid is approached the interchange of energy will become so rapid that it becomes meaningless to talk of lattice waves, since each component of instantaneous displacement will have changed shape while propagating through a distance of one wavelength ${ }^{30}$.

We will now extend the optical analogue of the Mössbauer effect to the case of increasing structural disorder.

For each particular solute molecule one would expect that the zero-phonon line should exist under all circumstances, but the background of transitions in which phonons are created or annihilated will tend to be reduced in width since the higher frequency part of the phonon spectrum separates out 
as localized vibrations as lattice periodicity is increasingly lost. In the limit of the amorphous solid each vibronic band of a particular solute molecule will consist only of a zero-phonon line and a few associated local vibrations of the solute molecule in the matrix cage. However the observed vibronic band widths for a disordered solid solution will not be those narrow band widths associated with an individual solute molecule since there will be a wide statistical distribution of solute-matrix interactions. For each vibronic band there will be a statistical spectrum of zero-phonon lines and of localized vibrations. The width of the vibronic band envelope will therefore depend on the variety of solute-matrix interaction configurations and the population of such configurations.

Experimentally one often observes a decrease in line widths on annealing a solid solution, in agreement with the above discussion, since such thermal treatment will give rise to a reduction in the number of intrinsic lattice defects, leading to increased periodicity of the lattice, and a concomitant greater homogeneity of solute molecule sites. The statistical plurality of solutematrix interactions in a defect solid therefore has an effect on vibronic band widths that much more than counteracts any reduction in the high frequency part of the phonon spectrum of the matrix due to loss of lattice periodicity.

In organic vitreous solutions, the vibronic band widths are of the order of $100 \mathrm{~cm}^{-1}$, or greater, which illustrates the great range of solute-matrix interactions in this case. It should be stressed that vibronic analyses based on bands of such width are to be considered with much caution, since such band envelopes may mask unresolved vibronic structure. An example of this is given in Figure 3 which compares broad bands of the fluorescence spectrum of benzene in E.P.A. glassy solution at $77^{\circ} \mathrm{K}$ with the well resolved structure of benzene fluorescence in crystalline cyclohexane solution at $77^{\circ} \mathrm{K}$.

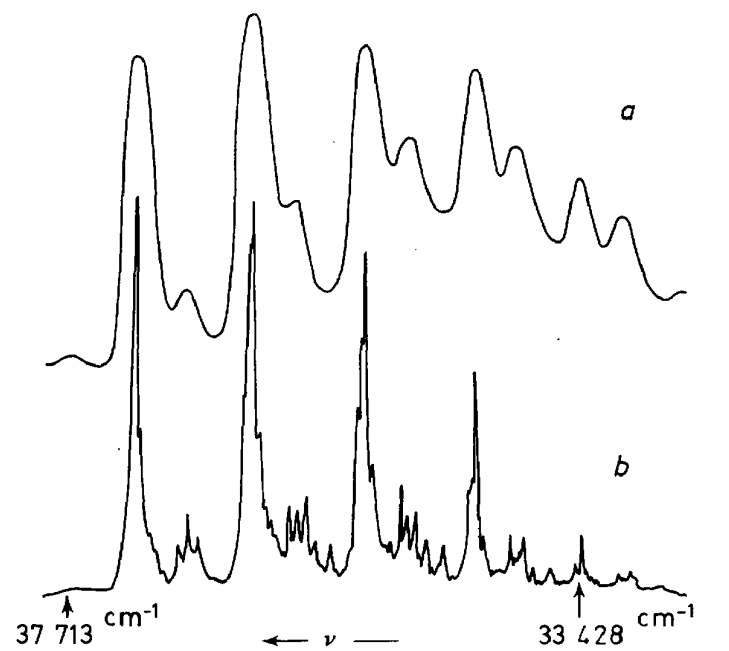

Figure 3. Fluorescence spectra of benzene in solid solution (concentration $10^{-3} \mathrm{M} / \mathrm{l}$ ) at $77^{\circ} \mathbf{K}$. (a) E.P.A. matrix. (b) Annealed cyclohexane matrix. The origin of spectrum $a$ has been shifted relative to spectrum $b$ to eliminate the difference in solvent shifts. The broad bands in spectrum $a$ are the envelopes of the peaks in spectrum $b$ each of which corresponds to an individual vibronic transition $^{52,53}$. 


\section{3. The dynamic variation of solute-matrix interactions}

The previous discussion has shown that the width and shape of a vibronic band will depend on the range of solute-matrix interactions. In the present section will be discussed the role of the dynamic variation of solute-matrix interactions which results from thermal vibrations of the matrix.

It is convenient to begin with the case of a completely disordered solid, to which a glass is a good approximation. This discussion will lead, inter alia, to an explanation of the apparent paradox that excitation of a glassy solution by monochromatic light gives rise to broad band fluorescence spectra.

In general, the solute molecule dimensions and interaction with its surrounding solvent cage will differ in the excited electronic state as compared with the ground state. Relaxation of the solvent cage, i.e. change of the equilibrium positions of the cage molecules, will in general not occur in low temperature solid solutions in times shorter than the fluorescent state lifetime which is of the order of $10^{-8} \mathrm{~s}$. (Relaxation could generally occur in times shorter than the phosphorescent lifetime, which can be of the order of one second for aromatic hydrocarbon solutes.) However, oscillations of the matrix molecules about their equilibrium positions have periods as short as $10^{-11}-10^{-12} \mathrm{~s}$. The vast majority of excited state molecules will therefore have been able to dissipate to the matrix any excess intramolecular vibrational energy before optical emission occurs. Fluorescence and phosphorescence emission in low temperature solid solutions will, in general, occur from the zero intramolecular vibrational state of the electronically excited solute. This will be true not only for glassy solutions but also for mixed crystals.

In a glass, the matrix consists of a set of coupled oscillators whose coupling constants have a wide statistical distribution. In durations of the order of $10^{-8} \mathrm{~s}$, because of the lack of translational invariance in the disordered medium, vibrations of the matrix due to thermal energy will cause the position of each matrix molecule (solute and solvent) to undergo a kind of local Brownian movement. The variation of the relative positions of the solvent cage molecules with respect to the solute molecule will create a time dependent modification of solute-solvent interactions. This can be considered as causing local, random, time dependent variations of the average dielectric constant of the medium.

Absorption spectra are obtained using a continuous light source or by sweeping the spectrum continuously. For each photon absorbed, the time involved $\left(\sim 10^{-15} \mathrm{~s}\right)$, in the interaction between the electromagnetic field and the absorbing molecule, will be much shorter than vibrational periods of the matrix so that the geometrical configuration of the solvent cage surrounding a particular absorbing solute molecule will be effectively 'frozen in' during the optical transition (Franck-Condon principle). If the absorption source were of picosecond duration, the resulting solute absorption spectrum would reflect the instantaneous spatial distribution of solute-solvent interactions range since the transition energy of a particular vibronic transition (zero phonon line plus local vibrations) would differ for each spatially different solute-solvent configuration.

However, the exposure times necessary for obtaining absorption spectra have hitherto been much greater than picoseconds (although the advent of high intensity mode-locked lasers makes picosecond absorption experiments 
possible ${ }^{31}$ ), so that in practice the absorption spectrum will correspond to an exploration of the time dependent variation of solute-solvent interactions among all possible solute sites. Since the range and relative 'population' of solute-solvent interactions will not vary with time, taking the solid solution as a whole, the range of solute-solvent interactions explored spatially by a picosecond source should be the same as that explored in normal sources over times which allow dynamic variation of solute-solvent interaction to take place.

Let us now consider the process of fluorescence excited by monochromatic radiation. Each absorbed monochromatic photon will select those solutesolvent cage local complexes which 'instantaneously' have energy levels in resonance with the incoming photon frequency. Since monochromatic excitation of picosecond duration is physically impossible because of the energy spread in such a short train of light waves, monochromatic excitation will, in practice, occur over durations permitting dynamic variation of solutesolvent interactions. The molecules excited by the incident monochromatic radiation in normal sources will therefore be those selected by temporal exploration of the dynamic variation of solute-solvent interactions. The excited molecules, as we have discussed above, will lose excess intramolecular (and local intermolecular) vibrational energy before emission. Furthermore, the relatively long radiative lifetime as compared with the matrix vibrational periods implies that, in general, the solute-matrix interaction for an individual solute molecule will be different at the time of emission as compared with that of absorption. The result is that, even with monochromatic excitation, the vibronic band widths for a glassy solution will be broad and reflect the full range and relative populations of solute-solvent configurations in the matrix. This discussion shows that the apparent paradox of narrow band excitation giving rise to broad band emission is a consequence of the dynamic vibrational properties of the glassy matrix and not of a static distribution of inhomogeneous equilibrium solute sites.

In more ordered solid solutions, the displacements of the lattice sites will generally have a smaller range than for disordered solids since, under a total potential energy of similar order of magnitude, the translational invariance in the former imposes concerted motion of the individual sites as compared with the Brownian motion type of displacement in the latter case. Therefore, the more perfect the solid, the narrower will be the range of dynamic variation of solute-matrix interactions and the sharper will be the vibronic band widths.

\section{SPECTROSCOPIC LINE WIDTHS IN ORGANIC SOLID SOLUTIONS: INFLUENCE OF SOLUTE SITE INHOMOGENEITY}

We have seen, in section III, that solute site inhomogeneities are undoubtedly an important factor determining vibronic band widths of the solute. For spectroscopic analysis it is required that line widths should be as narrow as possible so that vibronic band resolution is a maximum. The range of solute-matrix interactions and their dynamic variations must therefore be reduced to a minimum. This would be fulfilled for annealed true substitutional solid solutions.

Previous articles have discussed the physical conditions for the formation 
of substitutional organic solid solutions ${ }^{1,3}$. It was emphasized that for formation of substitutional organic mixed crystals, the solute and matrix molecules should have, ideally, the same molecular geometry and molecular volumes and that the interaction forces between solute and solvent molecules should be very similar in nature and strength to the forces operative in crystals of each species considered separately. Furthermore, for the formation of continuous solid solutions, the crystallographic system of solute should, in general (but with some specific exemptions), be identical with that of the solvent ${ }^{3,13}$.

It should be noted that these conditions are fulfilled exactly for isotopic mixed crystals, but in such cases there would be a strong interaction between the excited states of solute and solvent since they lie close to each other. As we have mentioned earlier, interest here is exclusively in cases where the solute and solvent excited states are sufficiently well apart in energy for interaction to be negligible, at least to first order ${ }^{3}$.

For such cases, the principal parameters investigated in spectral line width studies have been those of molecular geometry and molecular volume. Bolotnikova ${ }^{32}$ has studied the influence of the solvent on fluorescence line widths of naphthalene, anthracene and naphthacene in solid solutions at $77^{\circ} \mathrm{K}$. The matrices used were $n$-hexane, $n$-heptane, $n$-octane and $n$-nonane. She observed that the matrix giving the narrowest solute line width was that in which the length of the long axis of the fluorescent molecule was approximately the same as the length of the linear paraffin solvent molecule: naphthalene $7.2 \AA, n$-pentane $7.36 \AA$; anthracene $10 \AA, n$-heptane $10.0 \AA$; naphthacene $12.8 \AA, n$-nonane $12.8 \AA$.

It is probable that the solute molecule replaces a solvent molecule to form a substitutional solid solution in the most favoured cases or possibly, in the case of linear paraffin solvents, a kind of inclusion compound ${ }^{12}$. However it is not impossible that some of the solutions studied by Bolotnikova are in fact interstitial in nature ${ }^{26}$.

If the solute molecular volume is much bigger than the solvent molecule then defect structures or solute aggregation occurs. This has been found in our laboratory using cyclohexane as a solvent. Benzene, toluene, benzyl radical and fluorobenzene have molecular volumes which are less than or of the same order as that of cyclohexane $\left(\sim 100 \AA^{3}\right)$, so that lattice substitution in the matrix does not present steric problems. The vibronic band widths are less than $5 \mathrm{~cm}^{-1}$ for these solutes in annealed cyclohexane ${ }^{3}$ at $77^{\circ} \mathrm{K}$. However, polysubstituted benzene derivatives, whose molecular volumes are greater than that of cyclohexane, do not form substitutional solid solutions with this solvent. We have found that solute aggregation, and partial interstitial solution formation, occur at solute concentrations of $5 \times 10^{-2} \mathrm{M} / 1$ or $10^{-3} \mathrm{M} / 1$ for $o$-xylene ${ }^{33}, m$-xylene ${ }^{33}, p$-xylene ${ }^{33}$, mesitylene ${ }^{34}$, durene ${ }^{35}$, pentamethylbenzene ${ }^{36}$ and hexamethylbenzene ${ }^{37}$.

The electronic spectra of durene ${ }^{35}$, pentamethylbenzene ${ }^{36}$ and hexamethylbenzene ${ }^{37}$ in $n$-pentane, $n$-hexane and $n$-heptane solid solutions at $77^{\circ} \mathrm{K}$, at concentrations between $10^{-2} \mathrm{M} / 1$ and $10^{-4} \mathrm{M} / \mathrm{l}$, were found to be characteristic of the formation of interstitial solutions. Solute aggregation also occurred but to a decreasing extent in the more dilute solutions.

For the three solutes studied it was found that the band widths of the 
interstitial type solute spectra were most narrow in the $n$-hexane solid solutions. The range of solute-matrix interactions would therefore appear to be smallest in $n$-hexane solid solutions for these solutes.

On the basis of the results for naphthalene obtained by Bolotnikova ${ }^{32}$, durene, which resembles naphthalene in molecular size and in having $D_{2 h}$ symmetry, might have been expected to behave like naphthalene for which $n$-pentane is the best solvent. The fact that $n$-hexane is the best solvent for durene shows that a different parameter must be involved than in the case of naphthalene- $n$-pentane solid solutions. The long axis of durene is no greater than $7 \AA$ in length, according to the crystallographic data of Robertson ${ }^{38}$, whereas the $n$-hexane has a length of $8.57 \AA^{13}$. However, the diagonal axis formed by two opposed $\mathrm{C}-\mathrm{CH}_{3}$ bonds in durene is about $8.5 \AA$ in length. This axis, in the absence of mutual repulsion of neighbouring methyl groups, would be at an angle of $30^{\circ}$ with respect to the major axis of the aromatic molecule.

It was therefore suggested ${ }^{35}$ that for steric reasons durene molecules cannot fit into linear paraffin matrices in similar fashion to nonsubstituted linear aromatic hydrocarbons. The potential energy minimum for solutesolvent interaction is suggested to occur in $n$-hexane not when the long axis of the durene molecule is parallel to the $c$-axis of the matrix crystal (in the direction of which the solvent molecules are aligned), but when the solute molecule has rotated by $30^{\circ}$ about the $\mathrm{C}_{2}$ axis perpendicular to its molecular plane. Durene molecules will therefore tend to orient themselves in this fashion. The large band width observed, even in $n$-hexane, indicates that in interstitial solutions of this type, the matrix cages are highly irregular. A similar interpretation was given to account for $n$-hexane being the best solvent for pentamethylbenzene ${ }^{36}$ and hexamethylbenzene ${ }^{37}$.

Two more cases will be discussed in this section. The first concerns the observation of narrow line electronic spectra when the solute molecule is very much bigger than solvent molecules. Numerous examples have been studied by Shpolskii and his co-workers; indeed one of the earliest observations of narrow line spectra was for coronene in $n$-heptane. A striking case ${ }^{39}$ is that of phthalocyanine $\mathrm{C}_{32} \mathrm{H}_{18} \mathrm{~N}_{8}$ which gives sharp line fluorescence and absorption spectra in $n$-octane, $n$-nonane and $n$-decane at $77^{\circ} \mathrm{K}$. A solute molecule of this size must replace at least two solvent molecules and is probably trapped between layers of the paraffin molecules. Coupling with the matrix is evidenced by the fact that the coronene vibrational frequencies are solvent sensitive, varying by 1 to 2 per cent among the three paraffin solvents.

Narrow line spectra of phthalocyanine could be accounted for by considerations based on section III. 3. The presence of a solute of mass and size much greater than the matrix molecules could give rise to local vibrations where displacements of neighbouring oscillating lattice sites relative to the heavy solute are reduced as compared with their displacements in impurityfree regions of the paraffin crystal. The resulting narrow range of dynamic variation of solute-solvent interactions would give rise to narrow vibronic bands. Furthermore, the large number of matrix molecules in the solvent cage would tend to give a more smoothed out time dependent solute-solvent interaction. This interpretation is consistent with the fact that sharp line 


\section{ELECTRONIC SPECTRA OF ORGANIC SOLID SOLUTIONS}

electronic spectra are observed for coronene in three solvents of different linear dimensions ${ }^{13}: n$-octane $11.0 \AA$, $n$-nonane $12.8 \AA$, and $n$-decane $13.4 \AA$.

The other case of interest arises from the observation of broad vibronic bands in some substitutional solid solutions for which, on the contrary, one might expect narrow line spectra. An example is the fluorescence spectrum of $m$-xylyl radicals which have been formed by $\lambda 2537 \AA$ photolysis of $m$-xylene molecules in a $m$-xylene crystal ${ }^{33}$. The vibronic band widths are of the order of $300 \mathrm{~cm}^{-1}$ (Figure 4). The $m$-xylyl radicals may be formed by trapping of the $m$-xylene exciton at defect site complexes or, by analogy with observations on duryl formation in durene crystals ${ }^{40}$, via a single photon photosensitized reaction involving aromatic aldehyde impurities. In both cases $m$-xylene molecules atypically situated in the crystal lattice will be favoured sites for

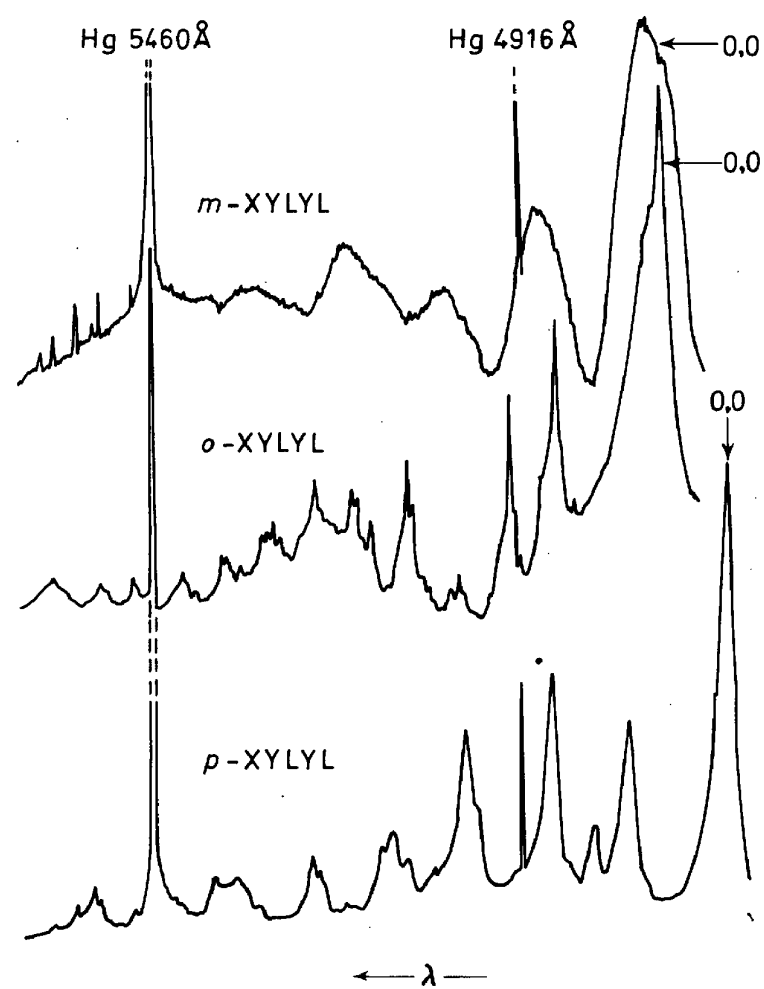

Figure 4. Fluorescence spectra at $77^{\circ} \mathrm{K}: m$-xylyl in polycrystalline $m$-xylene; $o$-xylyl in polycrystalline $o$-xylene; $p$-xylyl in polycrystalline $p$-xylene.

radical formation. A similar situation exists for formation of $o$-xylyl in $o$-xylene crystals and $p$-xylyl in $p$-xylene crystals. However, their vibronic line widths are much narrower, as can be seen in Figure 4.

The broadened bands of $m$-xylyl are not due to an intramolecular phenomenon since this radical, as well as $o$-xylyl and p-xylyl, give well resolved gas phase emission. spectra both at $\operatorname{low}^{41}$ and at high ${ }^{34}$ spectral 


\section{SYDNEY LEACH}

resolution. Furthermore, $m$-xylyl is known to give narrow line spectra in photolysed solutions of $m$-xylene in crystalline methylcyclopentane solid solutions ${ }^{42}$ at $88^{\circ} \mathrm{K}$. The width of $m$-xylyl fluorescence bands in $m$-xylene must therefore be due to a large range of radical sites, related to the range of sites of the photolysed parent molecules. It is possible that this larger range of radical sites as compared with $o$-xylyl and p-xylyl arises from the lesser hindrance to rotation of the methyl groups in $m$-xylene, leading to a greater and more varied number of defect sites being frozen in at low temperatures and an enhanced dynamic variation of radical-matrix interactions. The potential barrier to methyl rotation is $270 \mathrm{cal} / \mathrm{mol}$ for $m$-xylene, $1100 \mathrm{cal} / \mathrm{mol}$ for $p$-xylene and $2200 \mathrm{cal} / \mathrm{mol}$ for $o$-xylene ${ }^{43}$; it is interesting to note that this order is also that of decreasing line width for the fluorescence vibronic bands of these radicals.

\section{MULTIPLE SITES}

Absorption and fluorescence spectra of organic solid solutions often exhibit a multiplet structure i.e. each vibronic band of a spectrum has several components which repeat throughout the spectrum. Several years ago $^{44}$ we enumerated the variety of possible causes for this multiplet structure: multiple solute sites, lattice vibrations, matrix polymorphism, solute aggregates. Emphasis will be placed here on multiple solute sites and matrix polymorphism.

The work of Bowen and Brocklehurst ${ }^{45}$ and of Shpolskii and his associates $^{11,12}$ on the electronic spectra of coronene in linear paraffin solid solutions drew initial attention to the presence of line multiplets and their evolution with thermal treatment and purification of the solvent. The number of multiplets varies with the solvent ${ }^{12}$. These results and the more recent work of Kahane-Paillous and $\mathrm{Pfister}^{46,47}$ have shown clearly the existence of different solute sites in coronene-linear paraffin solutions. The nature of these sites is difficult to interpret but the geometrical factors involved have recently been discussed by Pfister ${ }^{47}$. Earlier it was suggested by Shpolskii and Klimova ${ }^{12}$ that frozen-in rotational isomers of the linear paraffins give rise to different solute sites.

The existence of frozen-in metastable forms of the matrix was demonstrated for quenched solutions of benzene in cyclohexane by Leach, Lopez-Delgado and Grajcar ${ }^{23}$. When the solutions were annealed the multiplet structure disappeared and only single vibronic lines were observed.

The fluorescence spectra of a $10^{-3} \mathrm{M} / 1$ solute of benzene in cyclohexane are shown, as a function of temperature, in Figure 5. For the solution initially quenched to $80^{\circ} \mathrm{K}$ from room temperature each vibronic band shows two components, HTT and BT, separated by $80 \mathrm{~cm}^{-1}$. The relative intensity of the HTT components decrease as the temperature is increased; the HTT components disappear completely at $175^{\circ} \mathrm{K}$ leaving only the BT components.

Cyclohexane undergoes a crystallographic phase transition at $186^{\circ} \mathrm{K}$ from the stable low temperature (BT) form II (whose structure is uncertain ${ }^{3}$ ) to the cubic structure, form I, stable at higher temperatures (HT).

It can be seen in Figure 5 that the HT spectrum above $186^{\circ} \mathrm{K}$ has broadened considerably and has shifted by about $100 \mathrm{~cm}^{-1}$ to higher energies with 
ELECTRONIC SPECTRA OF ORGANIC SOLID SOLUTIONS

respect to that observed at $175^{\circ} \mathrm{K}$. Reduction of the temperature below $186^{\circ} \mathrm{K}$ brings about a reverse change from the HT to the BT spectrum but the HTT components do not appear again. The HTT spectrum is therefore related to a metastable crystallographic form III of the cyclohexane matrix

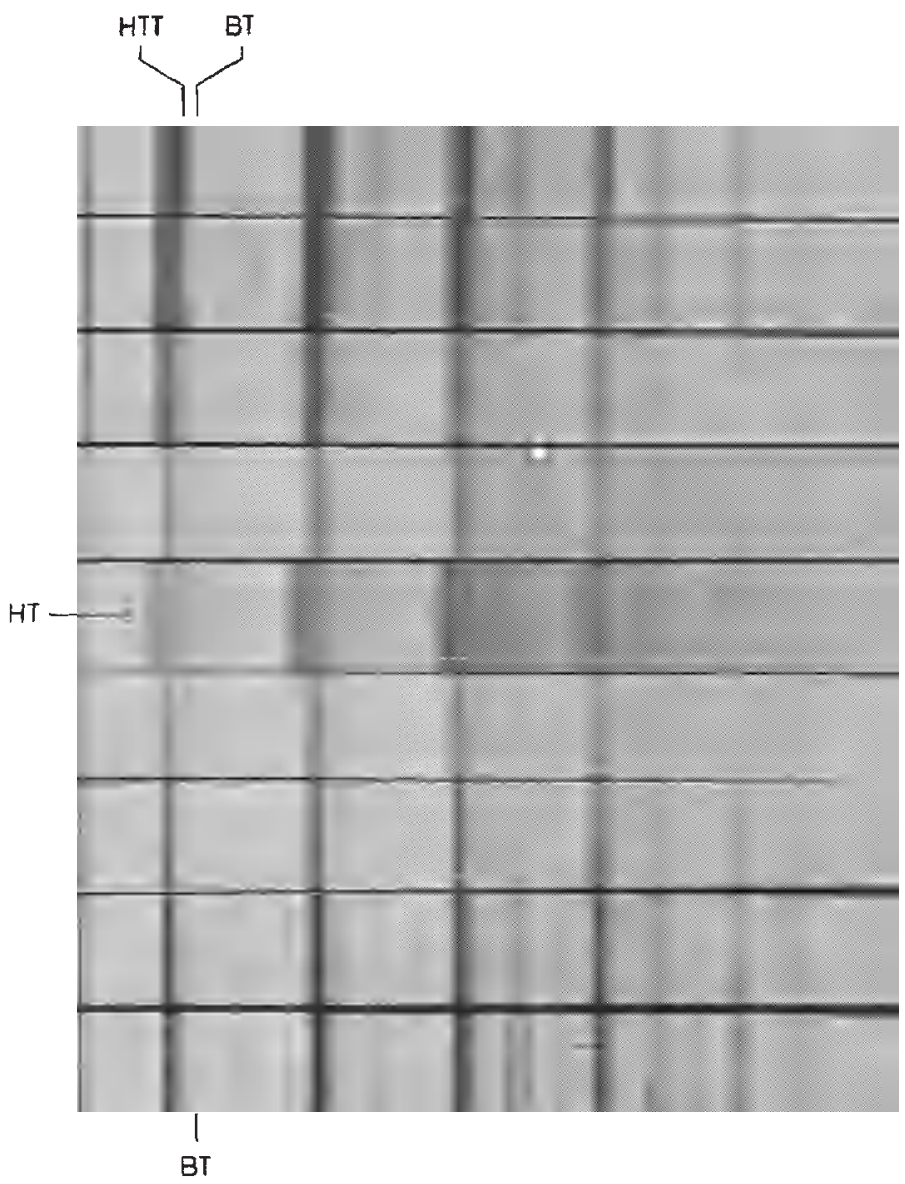

$T$ ㅇK

80

Figure 5. Effect of temperature cycle on the fluorescence spectrum of benzene in cyclohexane solid solution $\left(10^{-3} \mathrm{M} / 1\right)$.

whose formation is induced by the quenching operation. The BT and HT spectra are associated with forms II and I of solid cyclohexane respectively. Form I shows considerable rotational disorder ${ }^{48}$ and this could account for the broader vibronic bands above $186^{\circ} \mathrm{K}$.

The identification of solute sites can be carried out not only by thermal treatment experiments but also through the photo-excitation spectra of the fluorescence of solute molecules trapped at separate sites.

From the discussion in section III. 3 one can draw an important conclusion regarding such experiments. If solute-matrix configurations cannot be 
transformed into each other via vibrations of the matrix in times smaller than experimental times, then they will have separate excitation spectra. Such spectra have been observed for sites in the quenched cyclohexane solutions of benzene ${ }^{23}$ discussed above, in which each type of solute site, at $80^{\circ} \mathrm{K}$, is associated with individual microcrystals of different crystallographic structure, cyclohexane forms III and II, and which must be separated by grain boundaries hindering dynamic coupling between them.

Different excitation spectra of two components of the coronene fluorescence spectrum in $n$-hexane and $n$-heptane solid solutions were observed by Svishchyov ${ }^{49}$. As mentioned above, the nature of these sites is still uncertain.

In recent experiments in our laboratory, Michel $^{26}$ has studied the excitation spectra of the first three groups of multiplets of the fluorescence spectrum, at $3757 \AA(\alpha), 3788 \AA(\beta)$ and $3808 \AA(\gamma)$, of anthracene in $n$-heptane solution at $77^{\circ} \mathrm{K}$. The separation between $\alpha$ and $\gamma$ is about $390 \mathrm{~cm}^{-1}$ which is of the order of a $395 \mathrm{~cm}^{-1}$ low frequency vibration of anthracene. This led Bolotnikova et al..$^{50}$ to attribute the $\gamma$ multiplet band to the 0,0 transition and the $\alpha$ band to $0,0+390 \mathrm{~cm}^{-1}$, in which an upper state vibration is excited.

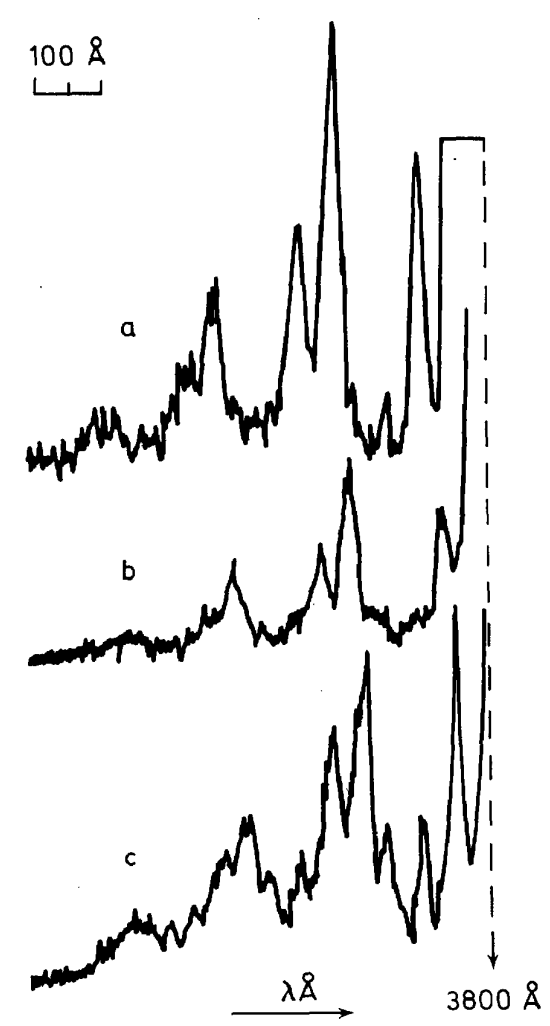

Figure 6. Excitation spectra of $\alpha, \beta$ and $\gamma$ components of the 0,0 region of the fluorescence spectrum of anthracene in quenched solid solution in $n$-heptane $\left(10^{-3} \mathrm{M} / \mathrm{l}\right)$ at $77^{\circ} \mathrm{K}$ :

(a) $\alpha$ component at $3757 \AA$; (b) $\beta$ component at $3788 \AA$; (c) $\gamma$ component at $3808 \AA$. 


\section{ELECTRONIC SPECTRA OF ORGANIC SOLID SOLUTIONS}

Such an interpretation implies that vibrational relaxation is incomplete in the excited electronic state. However Figure 6 shows clearly that the $\alpha, \beta$ and $\gamma$ bands have different excitation spectra, of similar allure but displaced about $25 \AA$ from each other, so that they must be attributed to 0,0 transitions associated with anthracene molecules in three different sites in the $n$-heptane matrix. Upper state vibrational relaxation is therefore normally effected in the $n$-heptane solution at $77^{\circ} \mathrm{K}$. Comparison of band intensities in absorption and fluorescence spectra showed however that the $\gamma$ band is a superposition of the $\gamma$ site 0,0 band and the $0,0-395 \mathrm{~cm}^{-1}$ band of the $\alpha$ site, the latter band involving a vibration excited in the ground state. This work will be reported more fully elsewhere.

In conclusion we wish to make a few remarks concerning the origin of multiple sites in terms of the mechanism of mixed crystal growth.

It is reasonable to suppose that for molecular solids, in which intermolecular forces are weak and generally non-directional, the presence of extrinsic impurities, whether they be the chosen solute molecules or accidental extraneous impurities, can induce particular and varied forms of short range or long range order in the surrounding matrix. In other words, in the presence of extrinsic impurities, the balance between minimum energy and maximum entropy determining the packing structure of the matrix around the solute can lead to a number of metastable minima of free energy and so to the co-existence of a number of different local structures in the polycrystalline medium. In some cases, the different structures may simply reflect the existence of misoriented solute or solvent molecules. The removal of impurities ${ }^{45}$ or the annealing of the solid ${ }^{46,47}$ would modify the number relative populations of these structures and so give rise to variations in the number of multiplets and their relative intensities. The persistence of multiple sites after thorough annealing of the solid solution indicates either that the potential barrier to the conversion of the different structures into the most stable one is very high or that the various minima of free energy have substantially the same value.

Finally it should be pointed out that the size of the microcrystals can be an important factor in determining the stable or metastable crystallographic form and the defect content. It is possible that in the initial nucleation stage a particular structural configuration of the incipient microcrystal could have a lower free energy than an equal volume of the equilibrium infinite crystal ${ }^{51}$. When growth has occurred beyond a certain critical size, the free energy will equal and then exceed that of an equal volume of the infinite crystal. The consequent strain energy will then be released by cleavage or by the introduction of dislocations and a crystallographic microphase transition to the new equilibrium structure. The growth to the final equilibrium infinite crystal could indeed take place via a series of different structural configurations having successively the lowest free energy of any possible configuration for that particular size of crystallite. It is therefore possible that in polycrystalline matrices, such as the linear paraffins, especially for quenched samples, the polycrystal could be composed of crystallites of different size having different structural configurations and different defect contents. This should be taken into account in interpreting the spectral observations of multiplet structure in solid solutions in linear paraffins. 


\section{SYDNEY LEACH}

\section{ACKNOWLEDGMENTS}

The author is indebted to many of his students and co-workers whose results are quoted here. Particular acknowledgment is due to Antonio Lopez-Campillo for helpful discussions during the preparation of this paper.

\section{References}

1 S. Leach. J. de Physique 28, C3-134 (1967).

2 S. Leach. Int. Conf. Spectroscopy, Bombay 1967, ICSU publication, (1968) p. 155.

3 S. Leach. 10.th European Congress on Molecular Spectroscopy, Liège 1969. Mem. Soc. Roy. Sci. Liège, $5^{e}$ sér., 20,179 (1970).

4 S. Leach, A. Lopez-Campillo, R. Lopez-Delgado and C. Tomas-Magos. C.R. Acad. Sci. (Paris) 263B, 1230 (1966).

5 (a) S. Leach and L. Grajcar. Fifth Int. Symp. Free Radicals, Uppsala, 1961, Almqvist and Wiksell, Stockholm (1961), paper 36;

(b) E. Migirdicyan and S. Leach. Bull. Soc. Chim. Belges. 71, 845 (1962).

6 J. von Kowalski. Phys. Z. 12, 956 (1911); Verh. deutsch. Phys. Ges. 13, 952 (1911).

7 G. N. Lewis and D. Lipkin. J. Am. Chem. Soc. 64, 2801 (1942).

8 I. Norman and G. Porter. Proc. Roy. Soc. (London) A230, 399 (1955).

9 S. Leach. Luminescence of organic and inorganic materials Ed. Kallmann and Spruch, Wiley, N.Y.(1962) p. 176.

10 D. S. McClure. J. Chem. Phys. 22, 1668 (1954); ibid. 24, 1 (1956).

11 E. V. Shpolskii, A. A. Il'ina and L. A. Klimova. Dokl. Akad. Nauk S.S.S.R. 87, 935 (1952).

12 E. V. Shopolskii. Soviet Phys. Usp. 3, 372 (1960); ibid. 5, 522 (1962); ibid. 6, 411 (1963).

13 A. I. Kitaigorodskii. Organic Chemical Crystallography, Consultants Bureau, N.Y. (1961).

14 A. I. Kitaigorodskii. Acta Cryst. 18, 585 (1965).

15 D. P. Craig, R. Mason, P. Pauling and D. P. Santry. Proc. Roy. Soc. (London) A286, 98 (1965).

16 J. D. Ferry. Viscoelastic properties of polymers Wiley, N.Y. (1961).

17 R. A. Swalin. Thermodynamics of solids Wiley, N.Y. (1962).

18 J. Sherwood. Organic solid state chemistry Ed. G. Adler, Gordon and Breach, N.Y.(1969) p. 37.

19 A. Bree and R. Zwarich. Mol. Crystals and Liquid Crystals 5, 369 (1969).

20 W. Helfrich and F. R. Lipsett. J. Chem. Phys. 43, 4368 (1965);

F. R. Lipsett and G. MacPherson. Can. J. Phys. 44, 1485 (1966).

$21 \mathrm{~S}$. Leach and E. Migirdicyan. Fluorescence à longue durée de vie de composés organiques, $9 \mathrm{e}$ série, Actions chimiques et biologiques des radiations. Ed. M. Haissinsky, Masson, Paris (1966) p. 117.

22 D. R. Scott and J. B. Allison. J. Phys. Chem. 66, 561 (1962).

${ }^{23}$ S. Leach, R. Lopez-Delgado and L. Grajcar. J. Chim. Phys. 63, 194 (1966).

24 R. Ostertag and H. C. Wolf. Phys. Stat. Sol. 31, 139 (1969).

25 C. Pfister. Private communication.

26 C. Michel. Thèse 3e cycle, Orsay 1970.

27 K. K. Rebane. Impurity spectra of solids Plenum Press, N.Y. (1970).

${ }^{28}$ S. Leach and R. Lopez-Delgado. J. de Physique Colloque $\mathrm{N}^{\circ} 2,139$ (1966).

${ }^{29}$ S. Leach and R. Lopez-Delgado. J. de Physique 28, C3-150 (1967).

30 P. G. Klemens, in Non-Crystalline Solids. Ed. V. D. Fréchette, Wiley, N.Y. (1960) p. 508.

31 P. M. Rentzepis. Photochem. Photobiol. 8, 579 (1968).

32 T. N. Bolotnikova. Opt. Spectr. 7, 138 (1959).

${ }^{33}$ S. Leach, A. Lopez-Campillo, R. Lopez-Delgado and M. C. Tomas-Magos. J. de Physique, 28, C3-147 (1967).

34 M. C. Cossart-Magos. Unpublished work in this laboratory.

35 G. Durocher and S. Leach. J. Chim. Phys. 66, 628 (1969).

36 G. Durocher. J. Chim. Phys. 66, 985 (1969).

${ }^{37}$ G. Durocher. J. Chim. Phys. 66, 637 (1969),

38 J. M. Robertson. Proc. Roy. Soc. (London) A141, 594 (1933); ibid. A142, 659 (1933).

39 F. F. Litvin and R. I. Personov. Sov. Phys. Doklady 6, 134 (1961);

R. I. Personov. Opt. Spectr. 15, 30 (1963).

40 E. Migirdicyan. In press, J. Chem. Phys. 55 (15 August 1971).

41 T. F. Bindley, A. T. Watts and S. Walker. Trans. Faraday Soc. 58, 849 (1962). 


\section{ELECTRONIC SPECTRA OF ORGANIC SOLID SOLUTIONS}

42 A. Pellois. Thèse 3e cycle, Rennes (1969);

A. Pellois and J. Ripoche. Spectrochim. Acta 26A, 1051 (1970).

43 J. Haupt and W. Muller-Warmuth. Z. Naturforsch. 23a, 208 (1968).

44 S. Leach. J. Chim. Phys. 61, 1629 (1964).

45 E. J. Bowen and B. Brocklehurst. J. Chem. Soc. p. 3875 (1954); ibid. p. 4320 (1955).

46 C. Pfister and J. Kahane-Paillous. J. Chim. Phys. 65, 876 (1968).

${ }^{47}$ C. Pfister. J. Chim. Phys. 67, 418 (1970).

48 M. Renaud and R. Fourme. J. Chim. Phys. 63, 27 (1966).

49 G. V. Svishchyov. Izv. Akad. Nauk S.S.S.R. 27, 696 (1963); Opt. Spectr. 18, 350 (1965).

${ }^{50}$ T. N. Bolotnikova, L. A. Klimova, G. N. Neresesova and L. F. Utkina. Opt. Spectr. 21, 237 (1966).

51 B. G. Bagley. J. Cryst. Growth 6, 323 (1970).

52 S. Leach and R. Lopez-Delgado. J. Chim. Phys. 61, 1636 (1964).

${ }^{53}$ R. Lopez-Delgado. Thèse d'Etat, Paris 1969 (C.N.R.S. n ${ }^{\circ}$ 3880). 